\title{
Adaptive Synchronization of Chaotic Systems with known Response System Parameters
}

\author{
Cheng Gan ${ }^{1, A}$, Yuan Cheng ${ }^{1, B}$, Ruiqi Wang ${ }^{2, C}$ and Junwei Lei ${ }^{3, D}$ \\ ${ }^{2}$ Department of Armament Engineering, Naval Aeronautical and Astronautical \\ University, Yantai Shandong 264001, China \\ ${ }^{3}$ Department of Control Engineering, Naval Aeronautical and Astronautical \\ University, Yantai Shandong 264001, China \\ ${ }^{A}$ gancheng@zzuli.edu.cn, ${ }^{B}$ chengyuan@zzuli.edu.cn, ${ }^{C}$ richkey1980@gmail.com, \\ Dleijunwei@126.com
}

\begin{abstract}
A kind of adaptive synchronous control method was proposed to solve a special synchronization problem between two chaotic systems, where the response system is totally known without uncertainty but the driven system contains both unknown parameters and uncertain nonlinear functions. An update law of estimation of unknown parameters of driven system by constructing a proper Lyapunov energy function and the stability of the whole system was guaranteed by Lyapunov stability theorem. What is worthy pointing out is that the chaotic systems are not required to satisfy the Lipscitz condition. At last, detailed numerical situation was done to show the rightness and effectiveness of the proposed method.
\end{abstract}

Keywords: Chaotic System; Synchronization; Adaptive Control; Nonlinear Function; Robustness

\section{Introduction}

Chaotic system has complex behavior and it is widely used in secure communication. Synchronization of two chaotic systems is a key technology of secure communication and that is why it attracted many researchers' interest. Rongwei Guo designed a kind of simple nonlinear adaptive control law to realize synchronization of two chaotic systems by using LaSalle theorem in paper [1]. It is a novel method but the unknown parameters and other uncertain situations are not considered. And the chaotic system are assumed to satisfy the so-called Lipscitz condition to guarantee the stability of the whole system. Although there are many chaotic system can be assumed to be bounded and satisfied the local Lipscitz condition. But the global Lipscitz condition is strict and many chaotic systems can not satisfy this global Lipscitz condition [2-9].

Since now most of the current synchronization are realized on the assumption that the chaotic systems used can satisfy the so-called global Lipscitz condition, Wei Lin [2] proposed a kind of adaptive synchronous strategy for chaotic systems can only satisfy the local Lipscitz condition or even the chaotic system is unbounded. But the unknown parameter and uncertain nonlinear functions are not considered simultaneously.

So in this paper, a kind of general chaotic system is taken as an example and the parameters of response system are assumed to be known, but the there are unknown parameters and uncertain nonlinear functions in driven chaotic system. A new kind of robust adaptive synchronous controller of uncertain chaotic systems was designed based on Lyapunov stability theorem. What is worthy pointing out is that the chaotic systems are not required to satisfy the Lipscitz condition. At last, detailed numerical situation was done to show the rightness and effectiveness of the proposed method [4-6]. 


\section{Problem Description}

Consider the below driven chaotic system and response chaotic system, where parameters of response system is known, the driven system with unknown parameters and nonlinear functions can be written as follows:

The driven chaotic system model can be written as

$$
\dot{x}=f_{x}(x)+F_{x}(x) \theta_{x}+\Delta(x, t)
$$

The response chaotic system model can be written as

$$
\dot{y}=f_{y}(y)+b u
$$

Taken a four dimension chaotic system as an example, the driven system can be expanded as

$$
\begin{aligned}
& \dot{x}_{1}=f_{x 1}\left(x_{1}, \cdots, x_{4}\right)+\sum_{j=1}^{p_{1}} F_{x 1 j}\left(x_{1}, \cdots, x_{4}\right) \theta_{x 1 j}+\sum_{j=1}^{p_{2}} \Delta_{x 1 j}(x, t) \\
& \dot{x}_{2}=f_{x 2}\left(x_{1}, \cdots, x_{4}\right)+\sum_{j=1}^{p_{1}} F_{x 2 j}\left(x_{1}, \cdots, x_{4}\right) \theta_{x 2 j}+\sum_{j=1}^{p_{2}} \Delta_{x i j}(x, t) \\
& \dot{x}_{3}=f_{x 3}\left(x_{1}, \cdots, x_{4}\right)+\sum_{j=1}^{p_{1}} F_{x 3 j}\left(x_{1}, \cdots, x_{4}\right) \theta_{x 3 j}+\sum_{j=1}^{p_{2}} \Delta_{x 2 j}(x, t) \\
& \dot{x}_{4}=f_{x 4}\left(x_{1}, \cdots, x_{4}\right)+\sum_{j=1}^{p_{1}} F_{x 4 j}\left(x_{1}, \cdots, x_{4}\right) \theta_{x 4 j}+\sum_{j=1}^{p_{2}} \Delta_{x 4 j}(x, t)
\end{aligned}
$$

And the slave response system can be expanded as

$$
\begin{aligned}
& \dot{y}_{1}=f_{y 1}\left(y_{1}, \cdots, y_{4}\right)+b_{1} u_{1} \\
& \dot{y}_{2}=f_{y 2}\left(y_{1}, \cdots, y_{4}\right)+b_{2} u_{2} \\
& \dot{y}_{3}=f_{y 3}\left(y_{1}, \cdots, y_{4}\right)+b_{3} u_{3} \\
& \dot{y}_{4}=f_{y 4}\left(y_{1}, \cdots, y_{4}\right)+b_{3} u_{4}
\end{aligned}
$$

where $\theta_{x}$ is unknown parameter and $\Delta_{x}$ is unknown nonlinear function, so the number of unknown parameters is $n * p_{1}$, and the number of nonlinear function is $n * p_{2}$ [7-9].

The objective of synchronization problem of chaotic system with unknown parameters and uncertain nonlinear functions is to design a control law $u=u(x, y, \hat{\theta}, \hat{q}), \dot{\hat{\theta}}=f(x, y, \hat{\theta}), \dot{\hat{q}}=f(x, y, \hat{q})$ such that the state of response system can track the state of driven system, then it means $y \rightarrow x$.

\section{Assumption}

Assumption 1: The response system has the same structure of the driven system and they have the same dimension $f_{x i}=f_{y i}$.

Assumption 2: Some parts of driven system are known, it means $F_{x i j}$ and $f_{x i}$ are known. 
Assumption 3: The response system is totally known, it means that $f_{y i}$ and $b_{i}$ are known.

Assumption 4: The nonlinear function of driven system satisfies following conditions. It means: for $1 \leq i \leq n, 1 \leq j \leq p_{2}$, there exists a unknown constant $q_{i j}{ }^{*} \leq d_{i j}$ such that

$$
\left|\Delta_{x i j}(X, t)\right| \leq q_{i j}^{*} \psi_{i j}(X)
$$

where $d_{i j}$ is a known constant and $\psi_{i j}(X)$ is a known positive smooth function [10$15]$.

\section{Design of Robust Adaptive Synchronization Controller}

Define an error variable as $z_{i}=y_{i}-x_{i}$, then the above drive - response system can be transformed as an error response of system as

$$
\begin{aligned}
& \dot{z}_{i}=f_{y i}\left(y_{1}, \cdots, y_{4}\right)-f_{x i}\left(x_{1}, \cdots, x_{4}\right) \\
& -\sum_{j=1}^{p_{1}} F_{x i j}\left(x_{1}, \cdots, x_{4}\right) \theta_{x i j}-\sum_{j=1}^{p_{2}} \Delta_{x i j}(x, t)+b_{i} u_{i}
\end{aligned}
$$

The control law can be designed as follows.

$$
\begin{aligned}
& u_{i}=f_{2 i}(x)\left[-f_{y i}\left(y_{1}, \cdots, y_{4}\right)+f_{x i}\left(x_{1}, \cdots, x_{4}\right)\right. \\
& \left.+\sum_{j=1}^{p_{1}} F_{x i j}\left(x_{1}, \cdots, x_{4}\right) \hat{\theta}_{x i j}+\sum_{j=1}^{p_{2}} \hat{q}_{i j} \psi_{i j}(x)-f_{z i}\left(z_{i}\right)\right]
\end{aligned}
$$

where

$$
\begin{gathered}
f_{2 i}(x)=b_{i}^{-1} \\
f_{z i}\left(z_{i}\right)=k_{i 1} z_{i}+k_{i 2} \frac{z_{i}}{\left|z_{i}\right|+\varepsilon_{i 1}}+k_{i 3} \frac{3}{2} z_{i}^{1 / 3} \exp \left(z_{i}^{2 / 3}\right)+k_{i 4} \operatorname{sign}\left(z_{i}\right)
\end{gathered}
$$

Then:

$$
z_{i} \dot{z}_{i}=z_{i}\left[-f_{z i}\left(z_{i}\right)-\sum_{j=1}^{p_{1}} F_{x i j}\left(x_{1}, \cdots, x_{4}\right) \tilde{\theta}_{x i j}+\sum_{j=1}^{p_{2}}\left\{\hat{q}_{i j} \psi_{i j}(x)-\Delta_{x i j}(x, t)\right\}\right]
$$

Where $\tilde{\theta}_{x i j}$ is defined as

$$
\tilde{\theta}_{x i j}=\theta_{x i j}-\hat{\theta}_{x i j}
$$

Considering that

$$
\begin{aligned}
& z_{i} \sum_{j=1}^{p_{2}}\left\{\hat{q}_{i j}{ }^{*} \psi_{i j}(x)-\Delta_{x i j}(x, t)\right\} \leq \sum_{j=1}^{p_{2}}\left\{z_{i} \hat{q}_{i j}{ }^{*} \psi_{i j}(x)+q_{i j}{ }^{*}\left|z_{i}\right| \psi_{i j}(x)\right\} \\
& =\sum_{j=1}^{p_{2}}\left\{\left|z_{i}\right| \psi_{i j}(x)\left[\operatorname{sign}\left(z_{i}\right) \hat{q}_{i j}+q_{i j}{ }^{*}\right]\right\}
\end{aligned}
$$

Define a new variable $\tilde{q}_{i j}$ as 


$$
\tilde{q}_{i j}=q_{i j}{ }^{*}+\operatorname{sign}\left(z_{i}\right) \hat{q}_{i j}
$$

Then it holds

$$
z_{i} \sum_{j=1}^{p_{2}}\left\{\hat{q}_{i j}^{*} \psi_{i j}(x)-\Delta_{x i j}(x, t)\right\} \leq \sum_{j=1}^{p_{2}}\left\{\left|z_{i}\right| \psi_{i j}(x) \tilde{q}_{i j}\right\}
$$

Considering that

$$
\dot{\tilde{\theta}}_{x i j}=\dot{\theta}_{x i j}-\dot{\hat{\theta}}_{x i j}=-\dot{\theta}_{x i j}
$$

Design the adaptive adjustment law as

$$
\dot{\hat{\theta}}_{x i j}=-z_{i} F_{x i j}\left(x_{1}, \cdots, x_{4}\right)
$$

In the same way, it is easy to get

$$
\dot{\tilde{q}}_{i j}=\operatorname{sign}\left(z_{i}\right) \dot{\hat{q}}_{i j}
$$

Design the adaptive adjustment law as

$$
\dot{\hat{q}}_{i j}=-z_{i} \psi_{i j}(x)
$$

Choose a Lyapunov function as

$$
V=\sum_{i=1}^{n} z_{i}^{2}+\sum_{i=1}^{n} \sum_{j=1}^{p_{1}} \frac{1}{2}\left(\tilde{\theta}_{x i j}\right)^{2}+\sum_{i=1}^{n} \sum_{j=1}^{p_{2}} \frac{1}{2}\left(\tilde{q}_{i j}\right)^{2}
$$

Solve its derivative and it is easy to get

$$
\dot{V} \leq \sum_{i=1}^{n}-z_{i} f_{z i}\left(z_{i}\right)<0
$$

According to the Lyapunov stability theorem [16-22], it is easy to prove that the system is stable and $z_{i}$ can converged to zero, then the synchronization is realized.

\section{5: The Analysis of Numerical Simulation}

Taking a four dimension hyper-chaotic system as an experiment object, the model of drive system is as follows.

$$
\begin{gathered}
\dot{x}_{1}=a\left(x_{2}-x_{1}\right)+k_{l b} x_{4} \cos x_{2} \\
\dot{x}_{2}=b x_{1}-k x_{1} x_{3}+k_{l b}\left(1+\sin \left(x_{2} x_{3}\right)\right) x_{2} \\
\dot{x}_{3}=-c x_{3}+h x_{1}^{2}+k_{l b}\left(2-\cos \left(x_{1} x_{2} x_{3} x_{4}\right)\right) x_{1} \\
\dot{x}_{4}=-d x_{1}+k_{l b} x_{3}\left(3+\sin \left(x_{1} x_{3}\right)\right)
\end{gathered}
$$

where $a, b, c, d$ are unknown parameters. The assumption conditions that uncertain nonlinear functions satisfy are the same as above. The structure of response system is known. Its model is shown as below: 


$$
\begin{aligned}
& \dot{y}_{1}=a_{y}\left(y_{2}-y_{1}\right)+u_{1} \\
& \dot{y}_{2}=b_{y} y_{1}-k y_{1} y_{3}+u_{2} \\
& \dot{y}_{3}=-c_{y} y_{3}+h y_{1}^{2}+u_{3} \\
& \dot{y}_{4}=-d_{y} y_{1}+u_{4}
\end{aligned}
$$

System parameters are set as $\left(a_{y}, b_{y}, c_{y}, d_{y}\right)=(9,39,2.4,-10.5)$. The initial states of drive system are set as $\left(x_{1}, x_{2}, x_{3}, x_{4}\right)=(1,-1,2,-2)$. And the initial states of response system are set as $\left(y_{1}, y_{2}, y_{3}, y_{4}\right)=(-3,3,-5,5)$.

The error of system is shown as below.

$$
\begin{gathered}
\dot{e}_{1}=a_{y}\left(y_{2}-y_{1}\right)-a\left(x_{2}-x_{1}\right)-k_{l b} x_{4} \cos x_{2}+u_{1} \\
\dot{e}_{2}=b_{y} y_{1}-k_{1} y_{1} y_{3}-\left\{b x_{1}-k_{1} x_{1} x_{3}+k_{l b}\left(1+\sin \left(x_{2} x_{3}\right)\right) x_{2}\right\}+u_{2} \\
\dot{e}_{3}=-c_{y} y_{3}+h y_{1}^{2}-\left\{-c x_{3}+h x_{1}^{2}+k_{l b}\left(2-\cos \left(x_{1} x_{2} x_{3} x_{4}\right)\right) x_{1}\right\}+u_{3} \\
\dot{e}_{4}=-d_{y} y_{1}-\left\{-d x_{1}+k_{l b} x_{3}\left(3+\sin \left(x_{1} x_{3}\right)\right)\right\}+u_{4}
\end{gathered}
$$

The designed control law is as follows

$$
\begin{aligned}
& u_{i}=\left[-f_{y i}\left(y_{1}, \cdots, y_{4}\right)+f_{x i}\left(x_{1}, \cdots, x_{4}\right)\right. \\
& \left.+\sum_{j=1}^{p_{1}} F_{x i j}\left(x_{1}, \cdots, x_{4}\right) \hat{\theta}_{x i j}+\sum_{j=1}^{p_{2}} \hat{q}_{i j} \psi_{i j}(x)-f_{z i}\left(z_{i}\right)\right]
\end{aligned}
$$

Definition and selection of controller parameters are shown as above. The simulation results are shown in following figures.

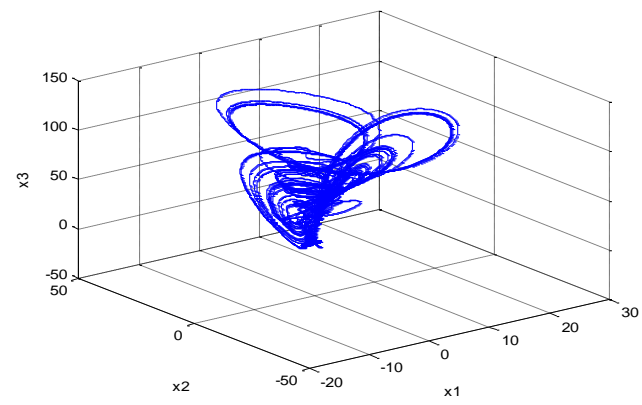

Figure 1. Trajectory of Uncontrolled Chaotic Sysmtes(1) 


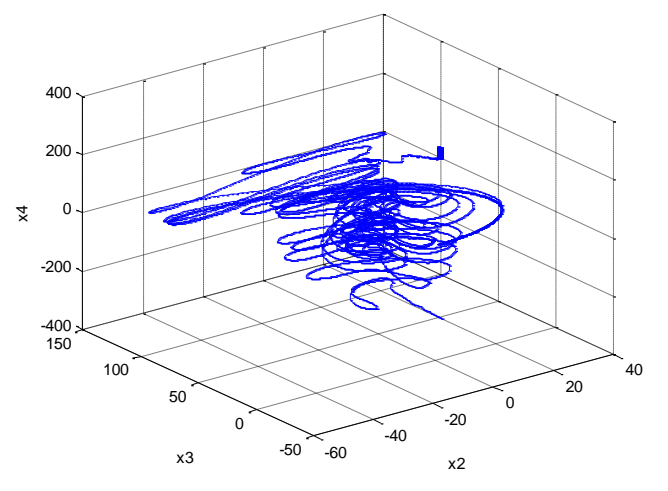

Figure 2. Trajectory of Uncontrolled Chaotic Sysmtes(2)

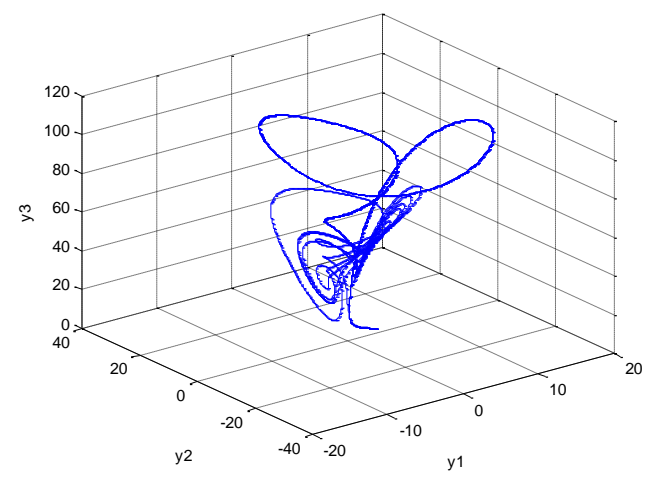

Figure 3. Trajectory of Uncontrolled Chaotic Sysmtes(3)

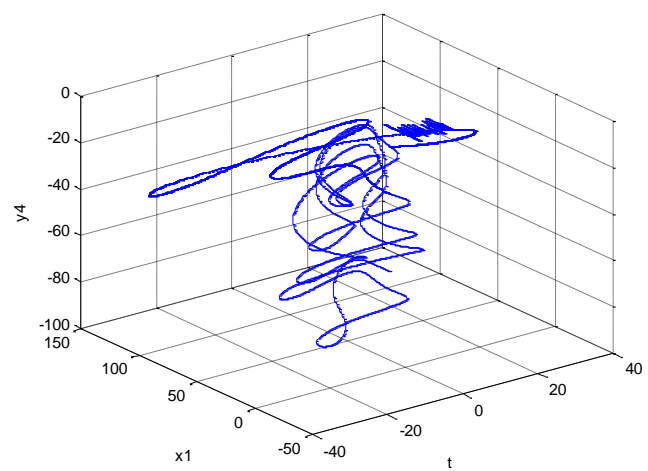

Figure 4. Trajectory of Uncontrolled Chaotic Sysmtes (4)

Figure 1 to Figure 4 shows the free movement trajectory of drive system and response system without control. Comparison of trajectory between drive system and response system without control can be shown from Figure 5 to Figure 8. It is obvious that states of two systems cannot synchronize with each other. 


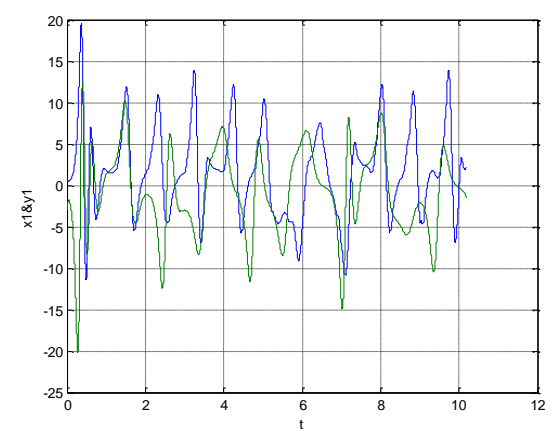

Figure 5. Trajectory of State $\mathrm{X} 1$ and $\mathrm{Y} 1$

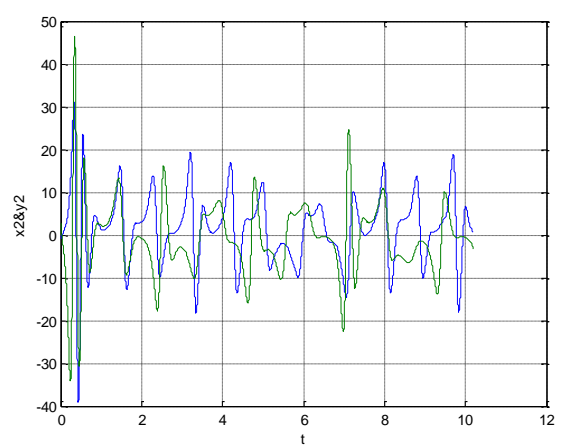

Figure 6. Trajectory of State X2 and Y2

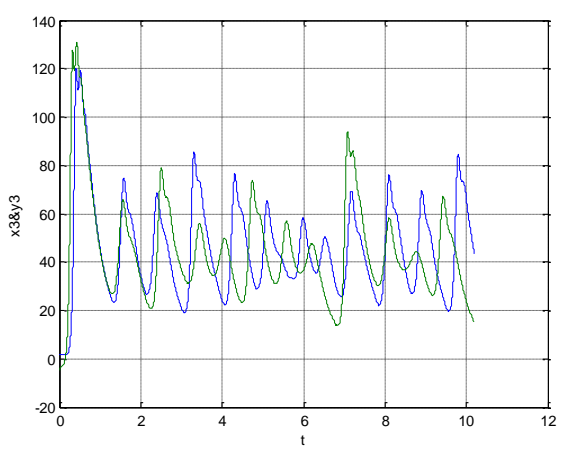

Figure 7. Trajectory of State X3 and Y3

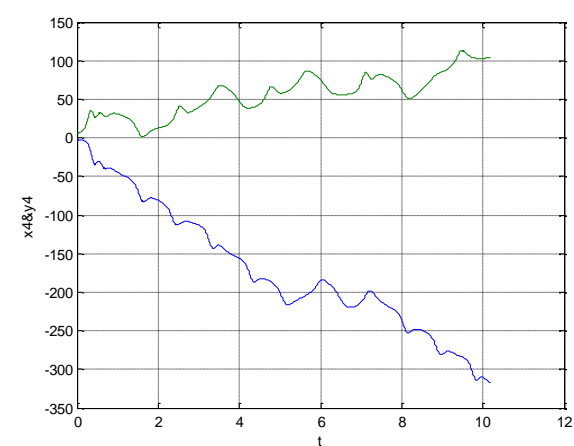

Figure 8. Trajectory of State X4 and Y4 
By adopting the proposed method of this paper, states of response chaotic system can track the state of driven system. The synchronization of each states can be shown from Figure 9 to Figure 12.

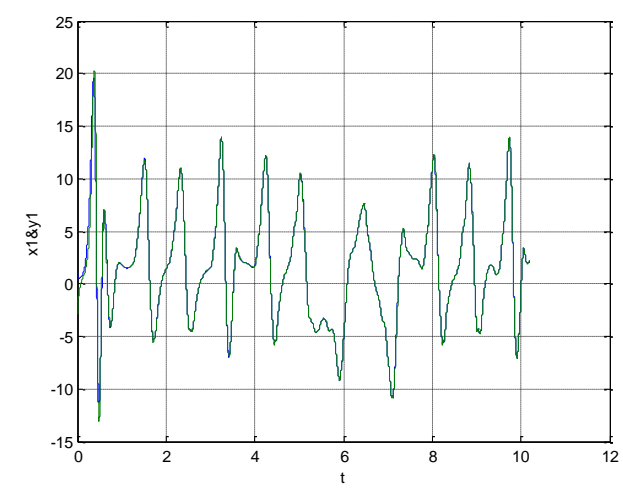

Figure 9. Tracing Curve of State X1

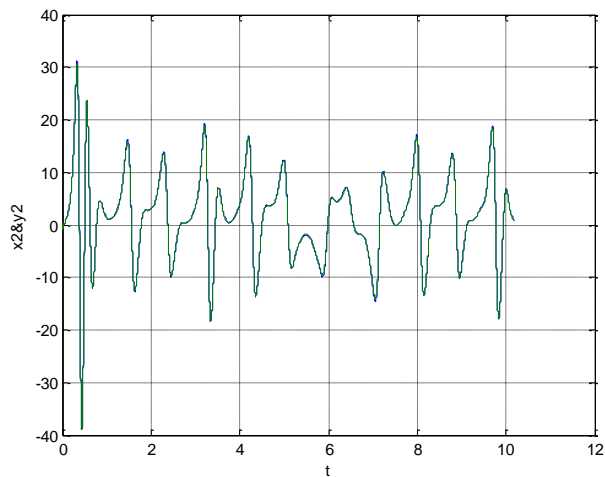

Figure 10. Tracing Curve of State X2

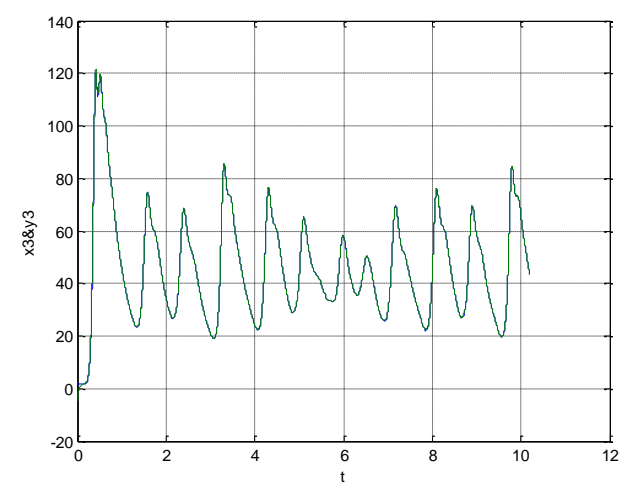

Figure 11. Tracing Curve of State $\mathrm{X} 3$ 


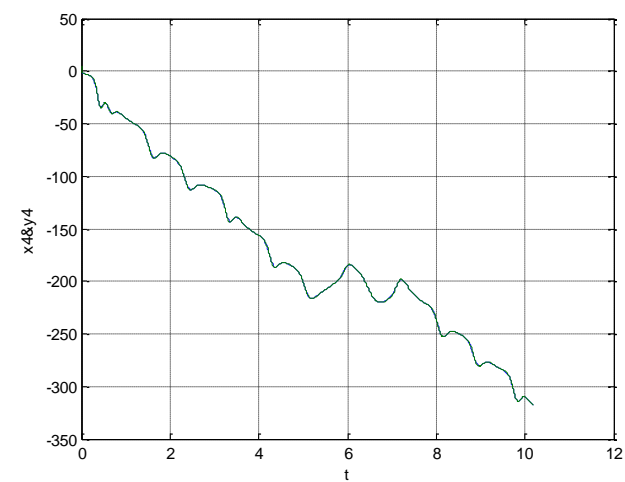

Figure 12. Tracing Curve of State X4

In summary, the drive system and response system can achieve fast synchronization by using method proposed in this paper for the drive system with unknown parameters and uncertain nonlinear functions.

\section{Conclusions}

A kind of robust adaptive synchronous strategy was proposed to solve the synchronization problem of chaotic systems. Uncertainties were considered and adaptive strategy was adopted to solve the unknown parameters. The update law was designed by constructing a Lyapunov energy function. And the bad affection caused by uncertain nonlinear functions were coped by robust method. At last, detailed numerical simulation were done to show the rightness of the proposed method.

\section{References}

[1] R. Guo, “A Simple Adaptive Controller for Chaos and Hyperchaos Synchronization”, Physics Letters A, vol. 360, (2009), pp. 38-53.

[2] W. Lin, "Adaptive Chaos Control and Synchronization in Only Locally Lipschitz Systems", Physics Letters A, vol. 372, (2008), pp. 3195-3200.

[3] N. F. Rulkov, M. M. Sushchik, L. S. Tsimring and H. D. I. Abarbanel, "Generalized Synchronization of Chaos in Directionally Coupled Chaotic Systems”, Phys. Rev. E 51, (1995), pp. 980-994.

[4] L. M. Pecora and T. L. Carroll, "Synchronization in Chaotic Systems", Phys Rev Lett., vol. 64, (1990), pp. 821-4.

[5] F. Liu, Y. Ren, X. Shan and Z. Qiu, "A Linear Feedback Synchronization Theorem for a Class of Chaotic Systems", Chaos, Solitons \& Fractals, vol. 13, (2002), pp. 723-30.

[6] M. Chen and Z. Han, "Controlling and Synchronizing Chaotic Genesio System Via Nonlinear Feedback Control", Chaos, Solitons \& Fractals, vol. 17, (2003), pp. 709-16.

[7] M. T. Yassen, "Adaptive Control and Synchronization of a Modified Chua's Circuit System", Appl Math Comput., vol. 135, no. 1, (2003) February 15, pp. 113-128.

[8] K. Y. Lian, P. Liu, T. S. Chiang and C. S. Chiu, "Adaptive Synchronization Design For Chaotic Systems Via A Scalar Driving Signal”, IEEE TransCirc Syst I, vol. 49, (2002), pp. 17-25.

[9] H. N. Agiza, "Chaos Synchronization of Lu Dynamical System", Nonlinear Anal TMA, vol. 58, (2004), pp. 11-20.

[10] J. H. Park and O. M. Kwon, "LMI Optimization Approach to Stabilization of Time-Delay Chaotic Systems", Chaos, Solitons \& Fractals, vol. 23, (2005), pp. 445-50.

[11] J. H. Lu, T. S. Zhou and S. C. Zhang, "Chaos Synchronization between Linearly Coupled Chaotic Systems", Chaos, Solitons \& Fractals, vol. 14, (2002), pp. 529-41.

[12] A. A. Alexeyev and V. D. Shalfeev, "Chaotic Synchronization of Mutually Coupled Generators with Frequency-Controlled Feedback Loop", Int J Bifurcat Chaos., vol. 5, (1995), pp. 551-7.

[13] M. Itoh, T. Yang and L. O. Chua, "Conditions For Impulsive Synchronization of Chaotic and Hyperchaotic Systems", Int J Bifurcat Chaos, vol. 11, (2001), pp. 551-6.

[14] R. Kilic, "Experimental Study on Impulsive Synchronization between Two Modified Chua's Circuits", Nonlinear Anal RWA, vol. 7, (2006), pp. 1298-303.

[15] J. H. Park, "Adaptive Synchronization of Hyperchaotic Chen System with Uncertain Parameters", Chaos, Solitons \& Fractals, vol. 26, (2005), pp. 959-64. 
[16] K. Grygiel, "Synchronization Of Two Coupled Second-Harmonic Generation Systems", Opt Commun., vol. 204, (2002), pp. 391-8.

[17] Y. Li, W. K. S. Tang and G. Chen, "Generating Hyperchaos Via State Feedback Control", International Journal of Bifurcat Chaos, vol. 15, (2005), pp. 3367-75.

[18] Z. Yan, "Controlling Hyperchaos in the New Hyperchaotic Chen System", Appl Math Comput, vol. 168, (2005), pp. 1239-50.

[19] K. Grygiel and P. Szlachetka, "Hyperchaos in Second-Harmonic Generation of Light", Opt Commun vol. 158, (1998), pp. 112-8.

[20] Y. W. Wang and Z. H. Guan, "Generalized Synchronization of Continuous Chaotic Systems", Chaos, Solitons, Fractals, vol. 27, (2006), pp. 97-101.

[21] Y. Wang, Z. H. Guan and H. O. Wang, "Feedback an Adaptive Control for the Synchronization of Chen System Via A Single Variable", Phys. Lett. A, vol. 312, (2003), pp. 34-40.

[22] X. Wu and J. Lu, "Parameter Identification and Backstepping Control of Uncertain Lü System", Chaos, Solitons, Fractals, vol. 18, (2003), pp. 721-729.

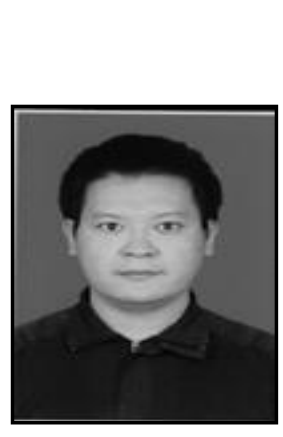

\section{Authors}

Cheng Gan (1981-) was born in Pingxiang city of Jiangxi province of China. He graduated from Zhengzhou University of Light Industry, Zhengzhou of China in 2002 and received bachelor's degree with the major of computer science and technoloy. He received his master's degree in 2006 from Huazhong University of Science and Technology and his major is computer application technology. He is now a lecturer in the Computer Department of Zhengzhou University of Light Industry. He published more than 10 academic papers, and 5 of them were retrieved by EI. His current research interests are the computer application technology and control theory.

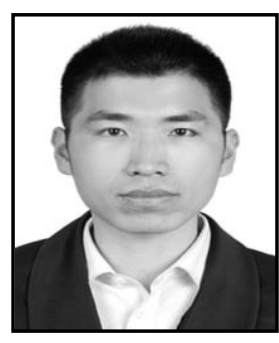

Ruiqi Wang (1980-) was born in Xinxiang City, Henan province of China. He received his Doctor degree in Guidance, Navigation and Control in 2011 from Naval Aeronautical and Astronautical University, Yantai of China. He graduated from Naval Aeronautical and Astronautical University, Yantai of China in 2001 and received his bachelor's degree with the major of missile control and test. After that he continued his study in this school and received his master's degree and doctor's degree in 2006 and 2011 respectively. He has published more than 20 papers, where 7 papers was indexed by Ei. His present interests are control theory, missile control and bilateral control.

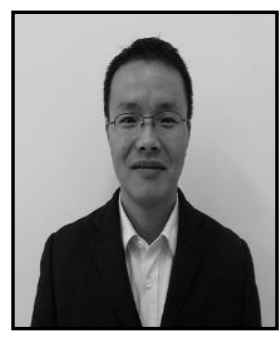

Junwei Lei (1981) was born in Chibi of Hubei province of China and received his Doctor degree in Guidance, Navigation and Control in 2010 from Naval Aeronautical and Astronautical University, Yantai of China. Her present interests are control theory, chaotic system control, aircraft control and adaptive control.

He was promoted to be a lecture of NAAU in 2010. His typical book named Nussbaum gain control technology of supersonic missiles was published in 2013 in China. 